\title{
Consistent contrast aids concept learning
}

\author{
DORRIT BILLMAN and DAVID DÁVILA \\ Georgia Institute of Technology, Atlanta, Georgia
}

\begin{abstract}
We suggest that coherence among concepts and correspondence between concepts and the world are important in concept learning. We identify one aspect of coherence, consistent contrast, and investigate its role in supervised concept learning. Concepts that contrast consistently carry information about the same attributes across the concepts within a contrast set. Concepts that contrast inconsistently predict and are predicted by values of different attributes. Experiment 1 revealed a large advantage for consistent contrast in learning and generalization. Experiment 2 pitted similarity against consistency and still revealed an advantage of consistency. Experiment 2 also broadened the range of tasks considered to include inductions about novel categories and subjects' category descriptions. We discuss relations to theories of concept learning, to attentional mechanism, and to alignability, and we suggest practical implications.
\end{abstract}

Some concepts are more sensible, natural, and useful than are others. What distinguishes the silly from the sensible? Some concepts are very easy to learn, others very difficult. What distinguishes the easy from the arduous? We suggest that two types of principles provide partial answers to both these questions: coherence and correspondence. By coherence, we mean consistent and extensive relations linking concepts together. By correspondence, we mean capturing predictive relations in the world. The importance of correspondence in concept structure is emphasized in Rosch's (1978) claims about the importance of capturing the correlational structure in the world and in research on learning correlations (within natural categories, Malt \& Smith, 1983; with or without labels, Billman, 1989, Billman \& Knutson, 1996, Cabrera \& Billman, 1996, Kersten \& Billman, 1997, and Medin, Altom, Edelson, \& Freko, 1982). The importance of coherence in concept structure is emphasized in research pointing to the importance of "theory" in guiding concept learning and use (Murphy \& Medin, 1985; Wisniewski \& Medin, 1994). When coherence and correspondence are directly considered, they are usually treated as conflicting or competing constraints (e.g., Vicente, 1990). We expect that relative importance will vary, and particular principles of either sort will sometimes conflict. However, we suggest that systems of concepts will in general be more easily learned and more useful once acquired when they are both highly coherent and correspond highly with relevant regularities in the world.

Part of this research was supported by National Science Foundation Grant 9732562. The authors thank Mike Firment for comments on a draft of this article. Partial results of Experiment 1 were presented at the Seventeenth Annual Conference of the Cognitive Science Society, 1995. D. Dávila is currently at weather.com, ddavila@mediaone.net. Correspondence should be addressed to D. Billman, Psychology Department, Jordan Hall, Stanford, CA 94303 (e-mail: billman@ @ psych. stanford.edu).
One coherence principle, consistent contrast (Billman, 1996), was investigated in the experiments reported here. We focused on how this principle facilitates concept learning but also examined how consistent contrast affects generalizations to new instances and inductions about new categories. We also assessed how this coherence principle affects the correspondence relations learned-specifically, knowledge of covariation among attributes.

In the introduction, we informally illustrate consistency of contrast, provide a more exact specification of consistent contrast, frame our work in the context of other investigations of coherence, and summarize our experimental approach. We provide our definitions of several key terms whose usage varies in the literature. Property is our general term, referring to any aspect (predicate, attribute, dimension, value, or feature) of an instance or category. Attribute refers to a variable-like property or dimension that can assume alternative values; it assumes nothing about the metric properties of the variable, but all attributes we use are categorical. Attribute value refers to a specific level or instance of the attribute. Color names an attribute; red names an attribute value. Category is used ambiguously to refer both to groupings in the world and to mental representations when these are assumed to be in relevant correspondence and to the grouping in the world only when external and internal structures diverge. Concept refers to mental representations - typically, of categories. Instance and example are used interchangeably to refer to an individual entity - typically, in the context of its membership in a category. Notice the parallelism between attributes (color) with their values (red) and categories (rabbit) with their instances (Peter).

\section{An Illustration of Consistent and Inconsistent Contrast}

When people, including children, think about animals, their knowledge is highly organized (Shipley, 1993). People know about many kinds of animals, and knowledge about one animal is coordinated with knowledge of other 
kinds. Specifically, the same sorts of properties-diet, vocalization, habitat, and so on - are important to and distinguish among many kinds of animals. Bears eat honey, growl, and live in caves. Monkeys eat bananas, chatter, and live in trees. Lions eat meat, roar, and live on the plains. People consider the same set of attributes relevant for characterizing and distinguishing these categories. These animal categories illustrate high "consistent contrast." Concepts with high consistent contrast may result from both selecting this organization from the world and imposing it even when it does not give the best fit.

Social role categories differ from animal categories in their contrast relations. The properties typical of one occupation seem to be rather different from those important for another. Personality and appearance, specifically, being extroverted and attractive, are important in our knowledge of what is generally true of actors. These attributes are irrelevant for doctors, who can be handsome or homely, but education and, perhaps, prosperity are important here. Furthermore, ranchers usually come from certain regions of the country and have land-holding families. The important attributes are highly varied: An attribute having homogeneous values for one role concept may have heterogeneous values for another. Category systems organized like this have inconsistent contrast.

We suggest that consistent contrast is an important coherence principle for conceptual structure. It would be fortunate if the structural principles that make concept learning easier also made the resulting concepts more useful. This might come about if our learning procedures are biased to discover useful categories, as suggested by various rational analyses (Anderson, 1991; Kornblith, 1995). We suggest that although the principles that make concepts easy to learn are not always the same as those that make the result useful, consistent contrast does play this dual role. Consistent contrast should aid learning because it helps localize what information should be used in discovery. Consistent contrast should aid use both because it should facilitate classifying novel instances into old categories and because it should facilitate reasoning about novel categories. This type of aid to induction was pointed out by Goodman (1954/1983) in his discussion of overhypotheses and has been exploited in both computational (Russell, 1986) and psychological (Macario, Shipley, \& Billman, 1990; Shipley, 1993) studies of induction. Given familiarity with animal kinds, on encountering one's first aardvark, one is appropriately biased to generalize diet but not dirtiness to other aardvarks. We predict that categories that contrast consistently will be learned more easily and support more reliable inductions and that such benefits can be found even when effects of instance similarity are controlled separately from consistency of contrast.

\section{Consistent Contrast}

In the present experiments, we investigated effects of coherence in the data by varying the consistency of contrast in novel categories. For a set of categories to contrast consistently, two background conditions must be met.
First, the set of categories must form a contrast set- that is, a set of alternative, mutually relevant categories. A good example is the set of mutually exclusive subordinate categories belonging to the same higher level category (see also Goldstone, 1996). Second, the categories in the set must be informative, not arbitrary collections of entities without predictive power about their properties. Since consistency of contrast measures how information is distributed, it is not relevant when categories do not convey information. To be informative, the probability density functions of attribute values within the categories must be nonuniform (within a category, some properties or combinations are more likely than others), and the probability density functions of the categories in the contrast set must be distinguishable (between categories, there is some difference). The categories have to be informative before we can ask how much of this information is consistently carried by the same attributes.

For informative, contrasting categories, consistency of contrast is high when information is carried by the same attributes across the contrast set, as in the illustration in which each type of animal has a distinctive type of diet. Contrast is inconsistent when information is carried by one set of attributes for one category and by another set of attributes for another, as in the illustration in which very different attributes are relevant to different occupational role categories. Consistency of contrast is a matter of degree, but, for simplicity, we will simply talk about consistent versus inconsistent contrast.

Categories might also differ in whether the same attribute takes on the same form of distribution (Martin \& Billman, 1991; Nisbett, Krantz, Jepson, \& Kunda, 1983). For example, each type of bird might take on one of two values of the color attribute. Consistency in the attribute's distribution would also increase consistency of contrast, but we did not investigate this here.

The idea of consistent contrast is most relevant when there are multiple, distinctive, alternative categories within a contrast set and multiple, distinctive, alternative values of the attributes. If a Category A were to be learned in isolation, it would have only a degenerate contrast set, of Category $\mathrm{A}$ and $\sim \mathrm{A}$; typically, there is no reason to expect negatively defined categories, such as $\sim \mathrm{A}$, to have any particular organization at all. If a property exists as an isolated predicate, uncoordinated with alternative values of one attribute, it can contribute only a modest degree of consistent contrast, since distinct but related alternative values are not available for distinct, alternative categories; the one property or predicate (perhaps "is magic" or "has a squiggly hook attached") only varies in degree of typicality for each category rather than assuming alternative values (see Garner, 1974, for related discussion). Indeed, a pressure for consistent contrast across clear alternatives may guide reorganization of isolated properties into coordinated attribute values. Concepts and properties differ in the extent and clarity of their contrast relations, and this graded nature of contrast will also produce graded degrees of consistency of contrast. 
In our experiments, we began the investigation of consistency of contrast by looking at the clearest cases. For the consistent contrast condition, attributes are either perfectly informative or carry no information at all about category membership. Furthermore, the same attributes are perfectly predictive across the contrast set. For the inconsistent contrast condition, different attributes are most informative for each category and each category has some perfectly predictable attributes. That is, consistent contrast and inconsistent contrast conditions differ in whether the informative attributes are identical across all contrasting categories or are completely different category to category.

\section{Consistent Contrast in Relation to Other Research}

One important aspect of coherence is the relation of new data to old theory (Ahn, 1999; Murphy \& Allopenna, 1994; Murphy \& Wisniewski, 1989; Rehder \& Hastie, 2001; Wisniewski \& Medin, 1994). However, the new data, or domain itself, can vary in the coherence or organization they afford. We suggest that the coherence of new data is a function of how well the concepts characterizing structure in the data can be related to each other, as well as to old knowledge. Consistent contrast is a coherence principle characterizing the organization afforded by a set of categories, whether within new data or spanning old and new categories.

Consistent contrast is similar to alignability (Gentner \& Markman, 1994) and to attribute systematicity (Barsalou \& Billman, 1989). All three specify higher order organizing relationships. Alignability and attribute systematicity apply at the level of instance pairs (alignability) or sets (attribute systematicity), and they index the degree to which the same attributes are specified across the instances. Instances with high attribute systematicity, or alignability, might have very similar ( $\operatorname{dog}$ and wolf) or very different (night and day) values on these common attributes. In work most related to the present research, Kaplan (1999) assessed the influence of alignability on ease of concept learning, using text stimuli, and found alignable pairs of categories easier to learn than nonalignable ones (see also Sifonis \& Ross, 1999).

Consistency of contrast applies at the level of categories, and it presupposes relatively high attribute systematicity/alignability at the instance comparison level. For example, in order for the attributes of diet and habitat to be informative across categories in a contrast set, they have to be specified for most instances in those categories. However, inconsistently contrasting categories can also have high alignability and attribute systematicity: Though the same attributes are specified across all instances (e.g., people), different attributes are relevant for different categories (e.g., ranchers vs. doctors).

In the present experiments, it was just this type of situation that we tested-equal, high attribute systematicity (or alignability) in conditions that contrasted either consistently or inconsistently. Thus, our experiments were not tests of alignability or attribute systematicity, since these are always high.

\section{Our Research}

The first experiment provided a basic demonstration of the effects of consistent contrast, whereas the second experiment pitted similarity against consistency. Our work focused on assessing principles (Billman, 1996; Kruschke, 1993; Medin \& Thau, 1992) rather than models, and we investigated a wider range of tasks than typically addressed by one model. We investigated the impact of the consistent contrast principle on several tasks, because we suspect that principles that influence learning will also influence concept use. We investigated speed of initial supervised learning, generalization of old categories to new instances, inductions about new categories, and category description.

Our work focused on the structure of a system of categories, rather than properties of an individual concept. Research in concept learning often focuses on a single category or discriminating one category from a single alternative. Investigating even a slightly more complicated conceptual system may allow us to clarify the role of a richer set of principles, important in much natural learning. We used three-category systems.

Our work focused on learning what information is relevant for a system of categories. When stimuli vary on two or three attributes and all variable attributes are relevant, the task requires setting criteria, not finding relevant information in a rich set of possibilities. Investigating slightly richer stimuli may tell us more about relevance learning, much needed in the world at large. Our stimuli varied on six attributes, two of which were relevant for a given category.

\section{EXPERIMENT 1}

In both consistent and inconsistent contrast conditions, the participants were presented with six-attribute alien animals from three categories and learned to classify them. In both conditions, there were two attribute values that were perfectly predicted by each category - that is, $p(\mathrm{~A} 1=1$, and $\mathrm{A} 2=1 \mid$ Category $=1)=1.0$. For example, in one configuration, Category 1 was the category of yodlars, A1 was the attribute of sound, A2 was the attribute of movement, and these attributes were perfectly predictable, since the probability of croaking and flying equaled one, given that the creature was a yodlar. Furthermore, in the consistent contrast condition, if sound and movement mattered for one category, they mattered for all three. However, in the inconsistent contrast condition, a different pair of attributes mattered for each category. We assessed classification during learning, and we also assessed what the participants had learned about the correlations between attribute values (e.g., between croaking sound and flying movement) in a final test.

\section{Method}

Participants. Fifty college students at the Georgia Institute of Technology received extra credit for participation. There were 27 
participants in the consistent and 23 participants in the inconsistent condition.

Stimuli. All stimuli were animated pictures of alien animals, created with Director (Macromind, 1992) for Macintosh. Each animal had six attributes (sound, movement, habitat, color, head, and limbs). Each attribute had three values. Figure 1 shows examples of the creatures. There were 45 learning trials, presenting 15 instances for each category. Each instance was displayed on the computer screen, with three buttons at the bottom of the screen labeled with the possible category names (yodlar, ralfaz, or muntog). Sets of instances were randomly generated for each participant from the set of possible instances consistent with the instance schemas in Table 1 and described below.

To control for the effects of salience of particular attributes, we used three different configurations. Different attributes were important in the different configurations. However, in all conditions and all configurations, some pair of attribute values mattered for each category; for example, all ralfazs lived in a desert habitat and were red in one configuration but made a yodeling sound and moved sideways in another. That is, for every category, there was some correlated pair of redundant relevant attributes values.

Condition (consistent vs. inconsistent) and conf iguration $(1,2,3)$ varied between participants. Abstract schemas for the learning items are shown in Table 1. Schema columns indicate attributes. Numbers indicate a specific value of the attribute, shared by all members of the category. Letters indicate randomly varying assignment of attribute values, which were randomized differently for each participant. The schema $x x 22 x x$ represents the ralfaz example above, because habitat and color are shown in the third and fourth columns of the schema, and red and desert are the second values of those attributes. Habitat and color are the relevant attributes for Category 2 (ralfaz) in both the consistent condition (in Configuration 2) and the inconsistent condition (in Configuration 1).

In the consistent contrast condition, the same attributes mattered for all three categories within a configuration, and choice of predictive attributes was counterbalanced across participants in the three configurations. In the inconsistent contrast condition, the same three pairings of attributes were used, but each category within a configuration used a different pair of attributes. Thus, within just one configuration, seen by one participant, each pair of attributes mattered for some category. For example, in Configuration 1, sound and movement were the important attributes for Category 1 , habitat and color for Category 2, and limbs and body/head for Category 3. Notice that the within-category structure was identical in the consistent and inconsistent contrast conditions. Attribute assignments were counterbalanced across configurations, ensuring that any condition differences could not be due to differences in attributes or attribute combinations.
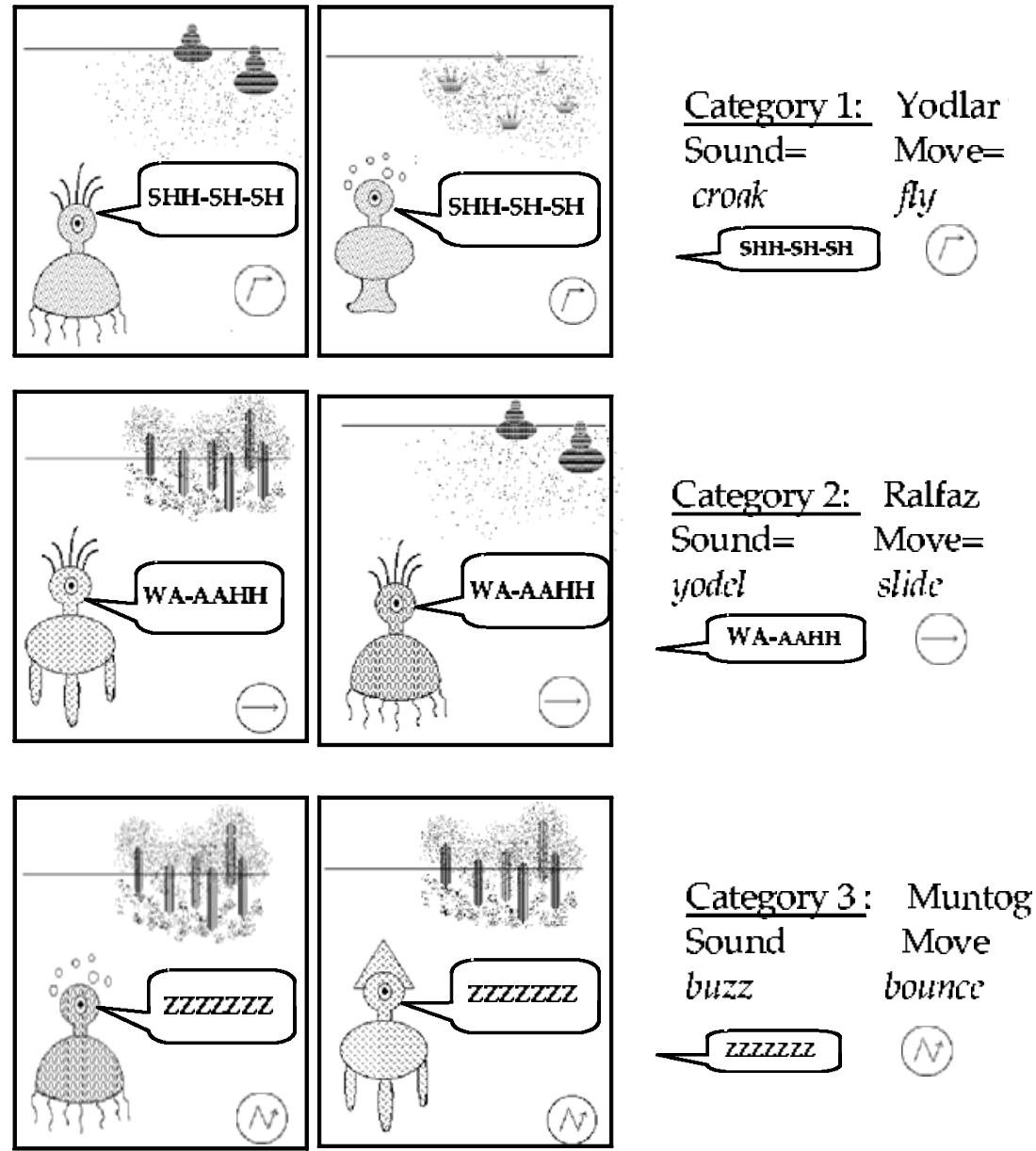

Figure 1. Examples of the learning stimuli from each of the three categories in Experiment 1 , Configuration 1 . The vocalization is printed in a balloon in the figure but was auditory for the participants. The motion is indicated by the arrow in the circle in the figure but was demonstrated by the animated figure moving across a larger screen area for the participants. 
Table 1

Abstract Schema for Consistent and Inconsistent Conditions of Experiment 1

\begin{tabular}{lccc}
\hline & $\begin{array}{c}\text { Category 1 } \\
\text { yodlar }\end{array}$ & $\begin{array}{c}\text { Category 2 } \\
\text { ralfaz }\end{array}$ & $\begin{array}{c}\text { Category 3 } \\
\text { muntog }\end{array}$ \\
\hline Consistent & & & \\
Configuration 1 & $11 x x x x$ & $22 x x x x$ & $33 x x x x$ \\
Configuration 2 & $x x 11 x x$ & $x x 22 x x$ & $x x 33 x x$ \\
Configuration 3 & $x x x x 11$ & $x x x x 22$ & $x x x x 33$ \\
Inconsistent & & & \\
Configuration 1 & $11 x x x x$ & $x x 22 x x$ & $x x x x 33$ \\
Configuration 2 & $x x 11 x x$ & $x x x x 22$ & $33 x x x x$ \\
Configuration 3 & $x x x x 11$ & $22 x x x x$ & $x x 33 x x$ \\
\hline
\end{tabular}

Note-Each cell represents the schema for a category within a condition (e.g., $11 x x x x$ ). Columns within the schema represent attributes: Column 1 = sound; Column 2 = movement; Column 3 = habitat; Column $4=$ color; Column 5 = head; Column $6=$ legs/body. Numbers represent specific, relevant attribute values, and $x$ s represent attribute values varying randomly within categories.

There were 30 test trials. The test assessed knowledge of the correlation between the two relevant attribute values for each category. Fifteen test instances were generated in the same manner as the learning instances and presented novel, correct items, 5 per category. Fifteen were generated by mispairing the attribute values that had gone together for each category during learning. For example, although correct Category 1 items had the $11 x x x x$ schema (e.g., move $=$ fly, and sound = croak), a $21 x x x x$ item might be shown as an incorrect test item (e.g., move $=$ slide, rather than move $=$ fly, but still with sound $=$ croak). The test trials required the participants to distinguish discrepant instances, which mispaired attribute values that co-occurred in learning instances, from those instances that correctly fit into any of the three categories seen at learning.

Procedure. The experiment consisted of learning, generalization, and debriefing phases. The participants provided their informed consent and were introduced to the task of learning about the animals in the Saturnian zoo. For both learning and test trials, a creature in a habitat vocalized and moved across the screen in a distinctive way. During learning, three buttons with category labels (yodlar, ralfaz, and muntog) were displayed at the bottom of the screen, the vocalizing animal moved across the screen, the participants clicked on the label they thought appropriate for the creature, and they were given feedback by the highlighting of the correct label. Initial chance performance could be improved by using category feedback.

The test phase assessed whether consistency of contrast influences learning about attribute correlations. Rather than continuing to classify items into the three old categories, the participants judged whether an item belonged in any of the categories they had seen during learning or did not fit and clicked a "yes" or "no" button. This fit/ misfit judgment is different from the classification task and thus is one type of transfer-of-knowledg e test, assessing what was learned in the process of learning to classify. This test is analogous to first teaching people about earth animals, including lions and eagles, and then asking them whether a misfit creature, such as a griffin, would fit in with the animals they had seen. No feedback was provided during the test phase.

The participants were debriefed about the nature of the experiment.

\section{Results and Discussion}

The participants in the consistent contrast condition scored much higher overall on learning trials (.858 correct, $S D=.099)$ than did the participants in the inconsistent contrast condition $(.494, S D=.088)$ [condition, $F(1,44)=$ $194.47, p<.001$; configuration, $F<1$ ]. Figure 2 shows that the consistent condition participants learned much more quickly and maintained their advantage through the learning phase. A repeated measures multivariate analysis of variance (MANOVA) by learning block found significant effects for condition $[F(1,48)=185.9]$, for learning block $[F(8,41)=17.76]$, and for their interaction $[F(8,41)=11.29$, all $p \mathrm{~s}<.001]$. On the last third of the learning trials (last three blocks), the participants in the consistent condition averaged .93 correct $(S D=$ $.111)$, whereas the participation in the inconsistent contrast condition averaged .52 correct $(S D=.181)$. On the last three blocks, 16 of 27 (59\%) of the consistent contrast participants scored perfectly but no inconsistent contrast participant did (significantly different proportions, $\left.\chi^{2}=26.19, p<.001\right)$.

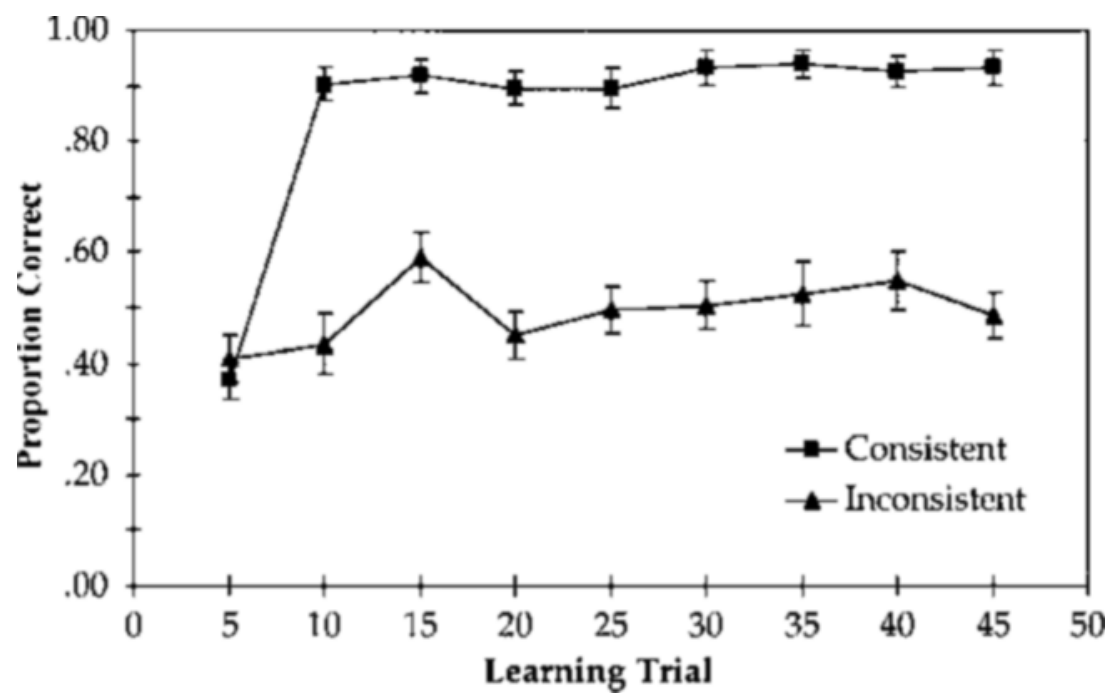

Figure 2. Experiment 1 learning curve, showing mean performance (and $S E M$ ) by groups of 5 trials in the consistent and inconsistent contrast conditions. 
The consistent condition participants were also much better at test. They showed more knowledge of correlations among attribute values, rejecting instances that had mispaired assignments of attribute values. Proportion correct in the consistent contrast condition was .743 $(S D=.251)$, higher than the inconsistent contrast proportion of $.5536(S D=.094)$ [condition, $F(1,44)=11.14$, $p=.002$; configuration within condition, $F<1]$, although test assumptions were not met. Distributions differed between conditions. In the inconsistent contrast condition, no participant consistently rejected mispairings (no score over .75), and $78 \%$ of the participants scored close to chance $(50 \%-60 \%$ correct inclusive; scores did average slightly but significantly above chance $[.95 \mathrm{CI}=.515$ to $.592]$ and were normally distributed). The consistent contrast condition scores were bimodal and nonnormal: Half the participants were consistently accurate in rejecting mispaired attribute values ( $48 \%$ of the participants scored $90 \%$ or higher), and the other half had scores scattered from .25 to .80 (suggesting idiosyncratic but less random responding than in the inconsistent condition). We scored the participants as learners or nonlearners, using a criterion of $90 \%$ correct. Nonparametric comparison of distributions shows significantly more learners in the consistent condition (13 of 27) than in the inconsistent condition $(0$ of 23$)\left(\chi^{2}=19.91, p<.001\right)$. (The difference is even larger if the learning criterion is reduced to $75 \%$.)

\section{EXPERIMENT 2}

Experiment 2 had two purposes. First, we sought effects of consistency on more aspects of category knowledge, including generalization to novel instances, sensitivity to correlations among properties, explicit category knowledge, and, particularly, inductions about novel, untaught categories. Experiment 1 demonstrated that consistent contrast aids learning initial categories and increases knowledge of covariation. It may also guide projection of that knowledge to novel situations, through overhypotheses. People use overhy potheses to guide inductions about novel categories within well-established category systems (e.g., Nisbett et al., 1983; Shipley, 1993), and we tested whether simply classifying instances on consistent attributes would be sufficient to change inductions about a new, untaught category. The induction task assessed whether the participants would selectively use the pair of relevant, correlated attributes to extend a novel category from one example. Since the participants in the inconsistent contrast condition had no consistently relevant attributes for the training categories, they would have no basis for forming overhypotheses to guide such inductions. Their performance provided a control condition for the consistent contrast participants. This comparison was a stronger control than comparison to chance: Systematic choice by the consistent contrast participants but not by the inconsistent contrast participants (on the identical items) ensured that the induction stimuli did not inadvertently cap- italize on any systematic, preexisting preferences. We also assessed the participants' explicit knowledge in a final participant report task.

Second, we controlled similarity, pitting similarity against consistency of contrast. Experiment 1 matched conditions on (1) all within-category similarity relations, (2) the number of categories, (3) the numbers of attributes, and (4) the numbers of attribute values. However, the between-category similarity relations were not equated. Generally, increasing consistency of contrast does increase the difference between categories. This is a necessary difference between extremely high and extremely low levels of consistency and was in fact a small but real difference in Experiment 1. In the inconsistent condition, randomly assigning each category-irrelevant attribute to any of its three values produced instances with attribute values typical of more than one category-for example, a Category 1 (croaks and flies, $11 x x \times x$ ) with other attributes typical of Category 2 (lives in the desert and red, $x x 22 x x$ ), such as 112231 . Such instances with conflicting cues made the categories in the inconsistent contrast condition more confusable or more similar to each other than were categories in the consistent contrast condition. Experiment 2 excluded these conflicting-cue instances in the inconsistent contrast condition, and it made between-category similarity lower (categories less confusable) in the inconsistent than in the consistent contrast condition.

\section{Method}

Participants. Thirty-four college students at the Georgia Institute of Technology received extra credit for participation. There were 17 participants in each condition.

Stimuli. Stimuli were very similar to those of Experiment 1, presenting three categories of alien animals distinguished by values on a pair of correlated, relevant attribute values. The same attributes were paired in a different manner from how they were paired in Experiment 1 , such that the most salient attributes were distributed more evenly: sound with color, movement with habitat, and head with legs. Categories were labeled zorack, lonkar, and shoosa. The schema design (Table 1) was the same as in Experiment 1 for the systematic attributes and for the attribute counterbalancing. Experiment 2 differed from Experiment 1 in the value assignments for the "random" irrelevant attributes: We excluded ambiguous values, and we controlled similarity.

A single set of 12 learning stimulus schemas was designed so that all pairwise similarity relations could be controlled. In particular, we ensured that multiplicative and additive similarity using a fixed metric favored the inconsistent condition (see the Appendix for details). We used 4 instances per category, presenting these 12 instances five times each over the course of learning. The exact attribute values for learning stimuli are shown in Table 2, with highly similar instance pairs indexed.

Two characteristics of these items are important for understanding the differences between Experiments 1 and 2 and between the two conditions of Experiment 2. First, no conflicting values were assigned to random attributes in the inconsistent contrast condition, a change from Experiment 1 . This change decreased the between-category similarity in the inconsistent contrast condition, since any instance in one category could match an instance in a different category only on 2 of the 6 attributes. That is, for all Category 2 items, A3 $=2$ and 
Table 2

Learning Stimuli for Experiment 2

\begin{tabular}{lccc}
\hline & Category 1 & Category 2 & Category 3 \\
\hline \multirow{4}{*}{ Consistent contrast } & \multicolumn{2}{c}{ Category Schema } & \\
Inconsistent contrast & $11---$ & $22---$ & $33---$ \\
& $11--$ & $--22--$ & ---33 \\
Consistent contrast & Learning Instances & \\
& 111111 & 222211 & $331312^{\mathrm{a}}$ \\
& $111312^{\mathrm{a}}$ & $223121^{\mathrm{b}}$ & $333322^{\mathrm{c}}$ \\
& $113121^{\mathrm{b}}$ & $222223^{\mathrm{d}}$ & $332223^{\mathrm{d}}$ \\
Inconsistent contrast & $113322^{\mathrm{c}}$ & $223233^{\mathrm{e}}$ & $333233^{\mathrm{e}}$ \\
& 111111 & 222211 & 221133 \\
& 111312 & 232212 & 231333 \\
& 113121 & 322221 & 323133 \\
& 113322 & 332222 & 333333 \\
\hline
\end{tabular}

Note-Schema columns represent attributes: Column $1=$ sound; Column $2=$ color; Column 3 = movement; Column 4 = habitat; Column 5 = head; Column $6=$ legs $/$ body. Numbers represent the values (1-3) used for each of the six attributes. Superscripts mark pairs of highly similar instances in different categories. Similarity favors the inconsistent contrast condition, with lower between-category confusability and greater within-category similarity.

A $4=2$, and these values were excluded from Category 1 instances (no red desert-dweller could also fly or croak); analogously, for all Category 1 items, A1 = 1 and A2 $=1$, values that were excluded from Category 2 (no flying, croaking animal could be red or live in the desert). Thus, a Category 1 item could match a Category 2 item only on Attributes 5 and 6 (head and body/legs). A side effect of reducing the between-category similarity in the inconsistent contrast condition was a change in how the predictive information was distributed across attributes. Each attribute was partly predictive for all categories in the inconsistent contrast condition. Consider Attribute 5: $p($ Category $=$ $1 \mid \mathrm{A} 5=1)=.50, p($ Category $=2 \mid \mathrm{A} 5=1)=.50$, and $p($ Category $=$ $3 \mid \mathrm{A} 5=1)=0$. In the inconsistent contrast condition, all attributes, even the "random" ones, carried some information about membership for all categories; this constraint was not imposed on the consistent contrast condition. As a result, (1) the consistency of contrast in the inconsistent contrast condition of Experiment 2 was greater than in the inconsistent contrast condition of Experiment 1, and (2) the difference in degree of consistency between inconsistent and consistent conditions is smaller in Experiment 2.

Second, attribute values were assigned to increase the betweencategory confusability of items in the consistent contrast condition relative to the inconsistent contrast condition, thus making betweencategory similarity work against any effect of consistency. Consider the second (11 13 12) to fourth (11 33 22) instances in Category 1 for the consistent contrast condition (Table 2). Each matched an instance in a contrasting category on the four last attribute values. However, no instance in the inconsistent contrast condition matched any instance in a contrasting category on more than two attribute values. Thus, the inconsistent contrast categories were less confusable with each other than were the consistent contrast categories. Both the within-categor y similarity relations and the betweencategory similarity relations favored the inconsistent contrast condition. Making similarity favor the inconsistent contrast condition was an important change introduced in Experiment 2.

Finally, controlling similarity requires controlling the salience of particular attributes and attribute values. As in Experiment 1, we did this by counterbalancing attribute assignment, so that each actual attribute (e.g., movement, color) played each schematically specified role equally often. Two orderings of learning and of test stimuli were used.

There were 24 test stimuli, half correct examples of the original three categories and half incorrect examples. The 12 correct test stim- uli were generated from the same schema as the learning items, 4 per category. The incorrect test stimuli, or "other" items, were generated by disrupting the pairs of attribute values that had covaried during learning. For example, for the consistent contrast condition, incorrect items used combinations of Attribute 1 and Attribute 2, such as 12 yy $z z, 31$ yy $z z, 23$ yy $z z$, rather than 11 yy zz, 22 yy $z z$, and $33 y y$ $z z$, whereas for the inconsistent contrast condition, incorrect items disrupted the 11 yy $z z, x x 22 z z$, and $x x$ yy 33 pairings.

The induction task introduced a novel category with a single source instance and then presented a series of animals for classif ication as belonging in that category or not. The participants saw a source instance (schematically, 444444 ), introducing a novel kind of animal also from the Saturnian zoo. All values on all attributes used in the induction task had never been seen during learning or generalization phases (schematically, Values 4-9), and, thus, all attribute values (as well as the instances they composed) were completely novel.

Two sets of induction problems were designed. Each had one source instance (44 4444 , or 7777 77) and six target instances. Half the targets matched the source on both relevant attributes (e.g., 4455 55 , or 445454 ), and we scored responses as correct if they included these items in the novel category. Half the targets matched on just one (e.g., 454555 ) or on neither of the relevant attributes (e.g., $\underline{55}$ 4455 ), and we scored responses as correct if they excluded these items from the novel category. The target instances varied the number of matching attributes (from 2 to 4 ), as well as which attributes matched. These stimuli allowed us to assess induction strategies based on similarity versus selective use of relevant attributes. The identical set and order of induction items were used for the consistent and inconsistent contrast participants within each configuration.

Procedure. The procedure was very similar to that of Experiment 1 , with the addition of an induction phase and extended participant questionnaire. Learning events were increased from 45 to 60 trials, but, otherwise, they were the same as in Experiment 1. The generalization phase procedure was changed from the two-alternative fit/not-fit judgments of Experiment 1 to four response alternatives (the three categories, plus "other"). The participants were told to respond "other" for instances that did not fit in with what they had seen during learning.

In the induction phase, the participants viewed the source instance, which was identified as belonging to a novel category, as many times as they wished. Then, they viewed six target instances. For each, they judged whether or not the target was the same kind as the source in- 
stance. The participants then viewed the second source instance and judged the second group of target instances. The participants in the consistent contrast condition who discovered that particular attribute(s) mattered for the previously learned categories of Saturnian animals might deem these attributes most relevant to membership in novel categories also. Induction choices consistent with these attributes were scored as contrast-based choices.

After the induction task, the participants filled out the participant report describing each of the three learning categories and then characterizing the two induction categories.

\section{Results and Discussion}

Learning. The consistent contrast condition participants learned faster and scored higher overall in the learning task ( .875 proportion correct, $S D=.157)$ than did the inconsistent contrast condition participants $(.763, S D=$ .125), even though between-category and within-category similarity favored the inconsistent contrast condition. By the end of learning, both groups were doing well, with $96 \%$ correct in the consistent condition and $89 \%$ in the inconsistent condition. Figure 3 shows the learning curves, with trials grouped into blocks of 5. A repeated measures MANOVA found significant effects of condition $[F(1,22)=$ $5.96, p=.023]$, of learning block $[F(11,12)=11.47, p<$ $.001]$, and of their interaction $[F(11,12)=3.12, p=$ $.031]$. There were no effects of configuration, of presentation order, or any of their interactions.

Generalization test. On the generalization test, the participants in the consistent contrast condition scored overall higher .67 proportion correct $(S D=.26)$ than did those in the inconsistent contrast condition at $(.50$ correct, $S D=$ .12) $[F(1,32)=6.07, p=.019]$. Recall that chance on this four-alternative test would be .25 . We divided the generalization items into the normal items and the correlationdisrupting "other" items. "Other" items measured knowledge of co-occurrence relations-for example, that old items might either be red (a zorack) or make a buzz sound (a lonkar), but never both were red and buzzed. The pattern of consistency advantage held both for the normal (.89 proportion correct in the consistent condition vs. 76 proportion correct in the inconsistent condition) and for the "other" items (.45 correct in the consistent condition vs. 24 correct in the inconsistent condition).

However, distributions for "other" items differed markedly between conditions, as shown in Figure 4. The consistent contrast condition distribution for "other" item scores was bimodal. Seven of 17 participants scored over $90 \%$ correct, showing that they learned the correlation and required the relevant properties to be properly paired. Six of 17 participants were correct on none of the "other" items and, indeed, never used the "other" response category at all, consistent with a single-attribute classification procedure. Since the consistent contrast distribution for "other" items was bimodal and nonnormal and it differed in form from the consistent contrast distribution, we compared proportion of high scores rather than central tendencies. Whether the individual performance criteria for success was set at $60 \%$ or $90 \%$, the numbers of successful correlation learners were 7 of 17 in the consistent contrast condition and 0 of 17 in the inconsistent contrast condition, a significant difference $\left(\chi^{2}=8.815, p=\right.$ $.003)$. The participants in the consistent contrast condition were more likely to learn and use attribute correlations, allowing them to correctly classify the discrepant "other" items. The results from participant protocols corroborate these findings.

Induction task. The participants in the consistent contrast condition selected induction items of novel categories based on the attributes relevant in their training categories more than expected by chance. They extended the novel categories to those instances matching on the attributes relevant in the consistent condition more than did the inconsistent contrast condition control participants. The par-

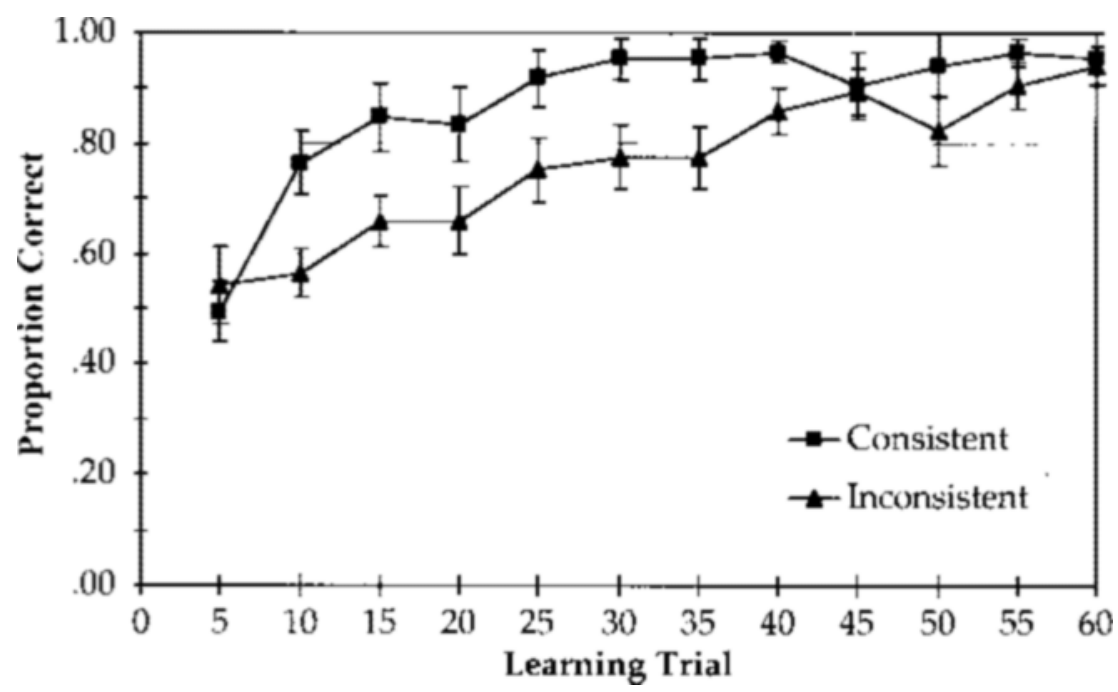

Figure 3. Experiment 2 learning curve, showing mean performance (and $S E M$ ) by groups of 5 trials in the consistent and inconsistent contrast conditions. 


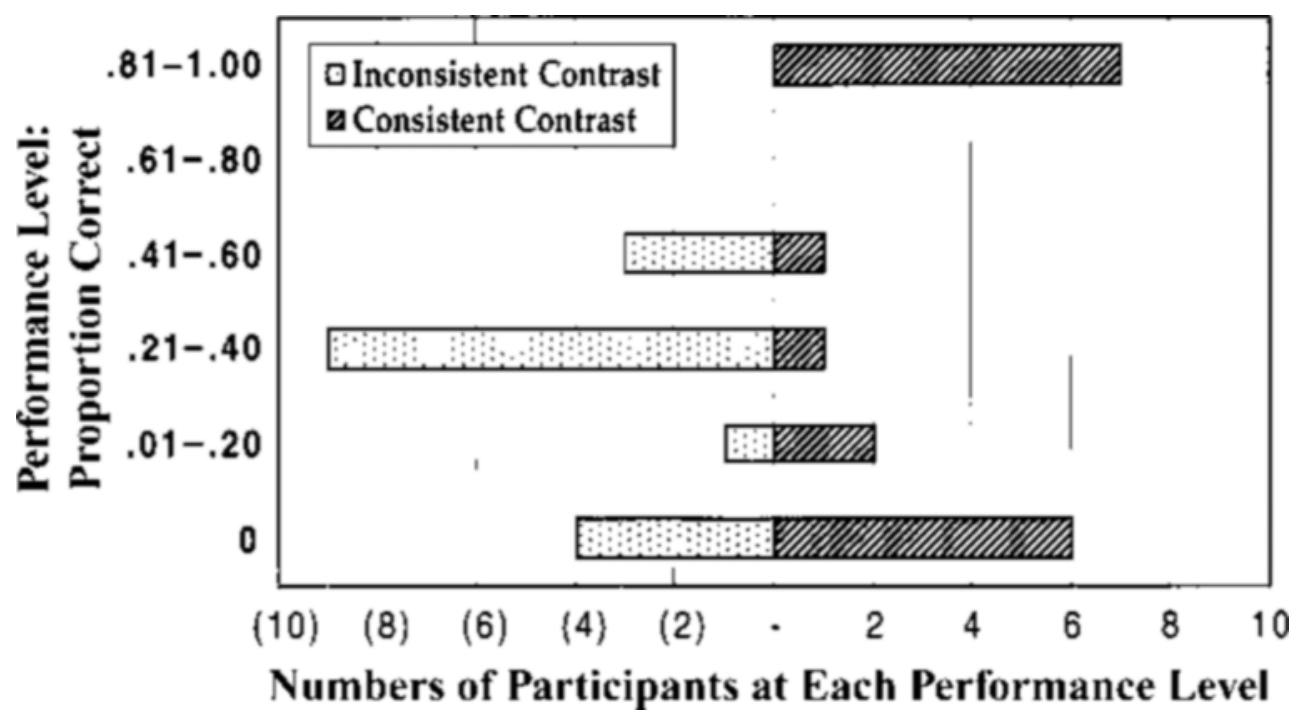

Figure 4. Frequencies of scores on "other" items in the generalization test in Experiment 2, shown by condition.

ticipants in the consistent contrast condition made target inductions $76 \%$ of the time $(S D=0.25)$, more often than chance $[t(16)=4.26, p<.001$, two tail $]$ and more than did the participants in the inconsistent contrast condition $(40 \%, S D=0.23)[F(1,32)=19.17, p<.001]$. Recall that relevance is consistently defined only for the consistent contrast condition, so the participants in the inconsistent contrast condition were not expected to prefer the "relevant" attributes. Indeed, the inconsistent contrast choices were marginally lower than chance $[t(16)=1.79, p=.09$, two tail]; this pattern would be expected if the inconsistent contrast participants (but not the consistent contrast ones) were choosing in accord with similarity, since, on average, the target inductions were somewhat less similar to the source instances than were the "incorrect" choices.

We also looked at individual judgment patterns to assess three different induction strategies: (1) similarity (number of matching attribute values between target and source predicts inductive generalization), (2) single attribute classification (match on one or the other of the two relevant attributes predicts inductive generalization), and (3) correlated attribute classification (matching both correlated, relevant attribute values predicts inductive generalization). Four correlations for each participant were computed, between item induction judgment and (1) total number of matching attributes, (2) match on first relevant attribute, (3) match on second relevant attribute, and (4) match on both relevant attributes.

Table 3 shows mean within-participantscorrelations; all four correlations differed between conditions. Specifically, the consistent condition participants selectively relied on one or both relevant attributes, indicating that they were not only learning about these attributes but were using them in inductions for a novel category. Furthermore, here the similarity index (number of matching attributes) cor- related negatively with inductive generalization. For the inconsistent contrast condition, there were fewer high correlations at either an individual or a group level, so judgments were much less systematic. However, inclusion judgments correlated positively with similarity and negatively with match on the relevant attributes, the opposite pattern from the consistent contrast condition.

Participant reports. The participants produced 169 characterizations (of old categories) that were scored for type, consistency, and accuracy. Description type codes the form of information: (1) reporting a single value of one attribute (e.g., "Lonkars are green"), (2) reporting a disjunction of attribute values for a category (e.g., "red or blue"), (3) specifying that all values of an attribute were acceptable (e.g., "can make any sound"), and (4) excluding specific values of an attribute (e.g., "not the bang-bang sound" or "not the Zorack motion"). For example, one inconsistent contrast participant's descriptions were "A Zorack had the bang-bang sound. A Lonkar just slid across the screen but didn't have the bang-bang sound. A Shoosa did not slide across the screen or have the bang-bang sound."

Participant consistency was scored for (1) whether a given attribute was used in descriptions of every category and (2) whether the identical set of attributes was used.

Table 3

Experiment 2 Induction Items: Average (and Standard Deviation) of Correlations Between Individual Participants' Responses and Item Characteristics

\begin{tabular}{lcrrr}
\hline & $\begin{array}{c}\text { Similarity: } \\
\text { No. of Matched } \\
\text { Attributes }\end{array}$ & $\begin{array}{c}\text { First } \\
\text { Relevant } \\
\text { Attribute }\end{array}$ & $\begin{array}{c}\text { Second } \\
\text { Relevant } \\
\text { Attribute }\end{array}$ & $\begin{array}{c}\text { Both } \\
\text { Relevant } \\
\text { Match }\end{array}$ \\
\hline Condition & $-.13(.33)$ & $.54(.50)$ & $.28(.40)$ & $.52(.52)$ \\
Inconsistent & $.23(.35)$ & $-.11(.48)$ & $-.28(.42)$ & $-.2(.49)$ \\
\hline
\end{tabular}

Note-All condition comparisons were significantly different at $p<.01$. 
Participant accuracy was also coded for (1) identifying at least one true, positive property for each category and (2) identifying both true positive properties available for each category.

Conditions differed in description type, accuracy, and consistency. Table 4 shows description type frequencies by individual (and by descriptions). Table 4 also provides $p$ values from $\chi^{2}$ or Fisher exact probabilities, as a guide to reliability. First, the forms of descriptions differed between conditions. In the consistent contrast condition, every participant for every category produced Type 1 (singlevalue) descriptions (e.g., "red," "makes the waah noise," or "cilla legs"), and these made up $90.9 \%$ of all descriptions. In the inconsistent contrast condition, only $39 \%$ of descriptions were of this single-value type. Recall that both conditions had the identical number of true properties for each category-properties that might have been equally included in descriptions from either condition. Nevertheless, the inconsistent contrast participants used the simple single-value forms less often and the more complicated forms of disjunction, negation, and attribute irrelevance more often. Furthermore, the participants in the inconsistent contrast condition used these forms to impose greater consistency of contrast.

Second, there was a striking finding regarding consistency: the imposition of consistency by the participants in the inconsistent condition. Consistency of participant descriptions were scored two ways: (1) for whether there existed any attribute which the participant used consistently across descriptions for all three categories and (2)

Table 4

Patterns in Form and Accuracy of Category Descriptions in Experiment 2

\begin{tabular}{lc}
$\begin{array}{l}\text { Consistent } \\
\text { Condition }\end{array}$ & $\begin{array}{c}\text { Inconsistent } \\
\text { Condition }\end{array}$ \\
\hline
\end{tabular}

Individual Category Descriptions by Type:

No. of Participants Ever Using Type (Total No. of Uses of Type)

Single Explicit Value ${ }^{1}$

17 (70)

$17(36)$

Value A or Value B

2 (4)

$0(0)$

$0(0)$

Excluded values

Total Uses

(77)

$9(20)^{* * *}$

$7(17)^{* * *}$

$9(14)^{* * * *}$

(92)

No. of Participants Reporting Designated Pattern for Set of Three Categories:

Consistency and Accuracy of Attribute Use across Category Set

One attribute the same

for all three categories

$17 \quad 9^{* *}$

Same set of attributes

for all three categories

14

$2 * * *$

One accurate value

for all three categories

14

$0^{* * * *}$

Both accurate, correlated values

for all three categories

5

$0^{*}$

Note-Multiple tests are not independent. Statistical comparisons are for number of participants, not number of uses. Likelihood ratios are used, or Fisher exact where expected cell $<5$. $\quad{ }^{1}$ Eight additional descriptions referred to unspecified single values, such as "had a certain sound"; these were similar to Type 1 responses but were scored separately. $* p<.05$. $* * p<.01$. ***p $<.001$. for whether the identical set of attributes was consistently used in descriptions for all three categories. Not surprisingly, the participants in the consistent condition produced more attribute-consistent descriptions (Table 4). Indeed, every consistent contrast participant used at least one attribute consistently across their descriptions of all three categories. What is remarkable, however, is that 9 of the 17 participants in the inconsistent condition also used at least one of the same attributes in their descriptions of all three categories. These descriptions were often accurate but less informative, as in specifying multiple allowable values of an attribute. Thus, the participants actively sought consistency in their descriptions, even when the domain structure clashed with this, resulting in either inaccurate or nonspecific descriptions.

Third, the consistent contrast participants provided more accurate properties. In the consistent contrast condition, every participant accurately identified a true attribute value (necessary and sufficient) for each of the three categories. No participant did this in the inconsistentcontrast condition, despite equal availability of such properties. The average numbers of relevant attribute values identified were 3.76 in the consistent condition and 1.65 in the inconsistent condition $[t(32)=4.55, p<.001]$.

Fourth, conditions differed in sensitivity to correlations - that is, noticing both correlated attribute values (redundant relevant cues) associated with one category. We scored the number of categories for which a participant reported the pair of correlated values ( $0-3$ categories). Five of the 17 participants in the consistent contrast condition accurately reported the two correlated values for each of the three categories, whereas no inconsistent contrast participant did. The average numbers of categories for which the participants reported both values were 1.06 in the consistent contrast condition (or one third of the time), and a mere 0.18 in the inconsistent contrast condition $[t(32)=2.52$, $p=.017]$. In the consistent contrast condition, this selfreport measure correlated strongly (.823) with our other measure of correlational knowledge, number correct on "other" items on the generalization test. The participants' self-reports (as well as the results of the generalization task) suggest better discovery of the co-occurring properties in the consistent condition than in the inconsistent condition.

\section{GENERAL DISCUSSION}

Experiments 1 and 2 established a benefit from consistent contrast on a variety of category tasks. The participants in the consistent contrast condition learned better, generalized better, and better transferred old knowledge to new categories. The participants were also more likely to learn correlations between relevant attribute values. The consistent contrast participants were better able to reject the "other" items, which required detecting items where the correlation was disrupted. They also were more likely to give category descriptions that identified the pair of correlated attribute values relevant for the category. 
A final piece of evidence for the importance of consistent contrast was the difference in form of the category descriptions-specifically, reduced reporting of the two properties predictive of and predicted by each category. The participants sought consistent use of the same attributes even when this led to more complex and less diagnostic descriptions of individual categories.

\section{Interpretation}

Two kinds of selectivity are important in learning: learning the attribute values associated with a specific category and learning the attributes relevant for the category set. Many participants sought and learned selective, specific rules, accurately linking attribute values and categories, as evidenced by category reports and errorless transfer on generalization. Many participants also sought and learned what attributes are relevant for this type of category, as evidenced by faster learning in the consistent contrast condition and performance on the induction task. Further support comes from the reports of the inconsistent condition participants who imposed consistency on the rules they discovered, even though this demanded rather peculiar rules for individual categories.

We propose that the two types of selectivity operate on different information and apply across a different scope. Attribute values are selected as relevant to particular categories (as in rules such as "Ralfazes are red"). Attributes themselves are selected as relevant to contrast sets ("color matters for characterizing the types of creatures"), producing the benefit of consistent contrast. Participants are linking specific attribute values with specific categories (as in rules) and important attributes with the contrast set (as in over-hypotheses; Goodman, 1983/1954; Macario et al., 1990; Martin \& Billman, 1991). As relevance of attributes is discovered, this guides which attribute values are preferentially used in rule learning; as successful rules are discovered, this adjusts the ratings of local attribute relevance, or the overhypotheses for the contrast set. This gives the two types of selectivity we found. (Notice that the distinction between attributes and attribute values can easily be masked if attributes are binary valued and only two categories are involved.)

In addition to "mutual aid" between categories, there is also mutual aid between attributes: The consistent contrast participants were more likely to use and report both correlated attributes for a category. Perhaps the greater coherence of the categories motivated the participants to seek further structure, looking for properties that could be predicted from the category, as well as searching for some property sufficient to categorize. This benefit from consistent contrast to learning correlations in supervised learning is strikingly analogous to the benefit from clustered features in unsupervised learning (Billman, 1989; Billman \& Knutson, 1996; Kersten \& Billman, 1997).

Our tasks also provide an important demonstration of flexibility across both stimuli and tasks. The knowledge learned from the initial stimuli was flexibly applied to novel stimuli, including stimuli that disrupted correlations seen during learning and stimuli with novel attribute values. The knowledge learned from classification was applied to other tasks, including induction from single instances of novel categories and providing descriptions of categories. This suggests that relatively flexible and abstract knowledge was acquired.

\section{Relation to Other Research in Concept Learning}

Our findings are sympathetic to the spirit of models that include selectivity at the level of both attributes and attribute values. Models such as Nosofsky and Palmeri's RULEX (Nosofsky \& Palmeri, 1998; Nosofsky, Palmeri, \& McKinley, 1994) and Erickson and Kruschke's (1998) ATRIUM include both rule learning and instance storage. These researchers have either implemented or suggested implementing attentional learning as part of the instance learning component. Considered this way, these models would provide for selectivity at the attribute value level in learning rules and selectivity at the attribute level in attentional learning for instances. However, both models isolate attentional learning from rule learning, keeping it within the instance component. When isolated in this way, attribute selectivity has no influence on the rule learning, as it seems to have had in our experiments. An older generation of rule-learning-plus-attentionmodels (e.g., Trabasso \& Bower, 1968) specifies selectivity at the attribute value level in rules and at the attribute level in attentional learning. Importantly, the attentional learning component in these accounts does modulate rule discovery, as happened in our experiments. The need for selectivity at the level of attributes (as in attention change) and attribute value (as in rule learning) is also noted in models of unsupervised learning (Billman \& Heit, 1988).

Two additional lines of research propose that selectivity operates at the level of both attributes and attribute values. Kersten, Goldstone, and Schaffert (1998) and Merriman (1999) propose distinct attentional change for attributes and for values. Our proposal differs from these in how and whether the scope of the change is localized. A localized change in selectivity means change in attention within a specific context and contrasts with a global change where the organism's use of or attention to information shifts in general, across stimuli and tasks. Merriman's proposal is most similar. He suggests that "dimensional dominance" is not a global shift but is based on importance in "similar situations." We also suggest that attribute importance is localized and specify that change is localized relative to the set(s) of contrasting categories.

Kersten et al. (1998) propose that attention changes globally at both attribute and value level. They suggest that once a given attribute value is associated with some other value, it will be less easily associated with other attribute values in general. We predict only local reduction in associability to a contrasting category, moderated by competing property-to-category links or rules, not general changes in associability of that one property to other properties in general. A global reduction in associability would imply reduced learning of relations to other correlated at- 
tributes. However, we found increased correlational learning in the consistent contrast condition, rather than in the inconsistent contrast condition, contrary to their prediction.

Least compatible with our findings are models that do not selectively alter the way data are used over the course of learning, at either the attribute or value level. Models in which all instances and attributes are used equally at the beginning and end of learning are not good accounts for our data. Specifically, those that do not specify how information use changes with learning are not good accounts of the phenomena we find (e.g., Anderson, 1991; Medin \& Schaffer, 1978).

Finally, can models with just one type of selectivity account for our data, or are two really needed? Anderson, Kline, and Beasely's (1979; Elio \& Anderson, 1981) rule learning model specifies selectivity at the level of the attribute values associated with specific categories but does not include any attentional learning. As a result, it will have difficulty accounting for consistent contrast effects. The generalized context models (e.g., ALCOVE; Kruschke, 1992; Nosofsky, 1984) include not only attentional or salience differences but systematic attentional change with concept learning, and these provide a selective mechanism at the level of attributes but not at the level of particular attribute values. ALCOVE is considered next in a bit more detail.

We asked whether ALCOVE would naturally predict the differences between the consistent and inconsistent contrast conditions. Billman and Dávila (1995) ran ALCOVE on the stimuli for Experiment 2, where similarity works against consistency. We extended the code provided by Kruschke to allow attentional change for multivalued categorical attributes. We used parameters selected by Kruschke (1990, Figures 2.3-2.10) to demonstrate the role of attentional learning, for stimuli of broadly similar nature and complexity. With these settings, the mean performance of ALCOVE was equivalent on consistent and inconsistent stimuli, showing no hint of an advantage for the consistent contrast condition. ALCOVE does not naturally predict the difference, but we did not attempt an exhaustive search of ALCOVE's very large parameter space. Perhaps sufficiently extreme attentional shifts could simulate the rule-like performance of our participants, at least in classification, without creating unwanted side effects. However, instance-plus-attribute representation and selectivity only at the level of attributes may be unsatisfactory because (1) participants report rules about values and (2) performance correlates with report.

Three traditions besides supervised concept learning also emphasize the importance of dynamic selectivity at the attribute level. In comparison of pairs of instances, the attributes used to interpret one stimulus are affected by a process of aligning the instance with a contrasting, comparison instance (Gentner \& Markman, 1994; Lassaline \& Murphy, 1998; Yamauchi \& Markman, 2000). In free sort tasks, rather than sequential, supervised learning, investigators have repeatedly found that adults and older children prefer to sort instances into a set of contrasting categories, relying on a consistent dimension (typically only one) for all categories (e.g., "intensional simplicity," Inhelder \& Piaget, 1964; see also Ahn \& Medin, 1992). This preference has been dubbed "1D sorting"; however, with stimuli typically used, it would be hard to distinguish whether participants are guided by a bias to use only one attribute/dimension or to use whatever set is chosen consistently across categories. In unsupervised learning tasks (with sequential presentation of instances and assessment of what is learned about stimulus structure), researchers have found that participants learn about attribute importance as well as about co-occurring attribute values (Billman, 1989; Billman \& Knutson, 1996; Clapper \& Bower, 1994; Kersten \& Billman, 1997).

\section{Significance}

For what scope of situations is the principle of consistent contrast relevant? For example, if structure in the world simply does not provide any consistency, this bias might be irrelevant in natural contexts. We suspect that much of the world does provide considerable, though partial consistency, in part because causal mechanisms in a domain are likely to produce systematic variation in a consistent set of attributes. Beyond this, however, we suspect that people will select and impose consistency even if not provided by the "raw" structure of the world, by adjusting categories, adjusting attributes, and satisficing accuracy. Finally, the principle may be relevant for designed environments and designed instruction about environments. Given alternative functional design possibilities, it may be desirable to select and construct those favoring consistent contrast.

\section{REFERENCES}

AHN, W.-K. (1999). Effect of causal structure on category construction. Memory \& Cognition, 27, 1008-1023.

Ahn, W.-K., \& Medin, D. L. (1992). A two-stage model of category construction. Cognitive Science, 16, 81-121.

Anderson, J. R. (1991). The adaptive nature of human categorization. Psychological Review, 98, 409-429.

Anderson, J. R., Kline, P. J., \& Beasely, C. M. (1979). A general learning theory and its application to schema abstraction. In G. H. Bower (Ed.), The psychology of learning and motivation (Vol. 13, pp. $277-$ 318). New York: Academic Press.

Barsalou, L. W, \& Billman, D. (1989). Systematicity and semantic ambiguity. In D. S. Gorfein (Ed.), Resolving semantic ambiguity (pp. 146-203). New York: Springer-Verlag.

Billman, D. (1989). Systems of correlations in rule and category learning: Use of structured input in learning syntactic categories. Language \& Cognitive Processes, 4. 127-155.

BILlman, D. (1996). Structural biases in concept learning: Influences from multiple functions. In D. L. Medin (Ed.), The psychology oflearning and motivation (Vol. 35, pp. 283-321). San Diego: Academic Press.

Billman, D., \& Dávila, D. (1995). Consistency is the hobgoblin of human minds: People care but concept learning models do not. In Program of the Seventeenth Annual Conference of the Cognitive Science Society (pp. 188-193). Hillsdale, NJ: Erlbaum.

Billman, D., \& Heit, E. (1988). Observational learning from internal feedback: A simulation of an adaptive learning method. Cognitive Science, 12, 587-625.

Billman, D., \& KNUTSON, J. (1996). Unsupervised concept learning and value systematicity: A complex whole aids learning the parts. Journal of Experimental Psychology: Learning, Memory, \& Cognition, 22, 458-475. 
Cabrera,A., \& Billman, D. (1996). Language-driven concept learning Deciphering "Jabberwocky." Journal of Experimental Psychology: Cognition, Learning, \& Memory, 22, 540-556.

Clapper, J. P., \& Bower, G. H. (1994). Category invention in unsupervised learning. Journal of Experimental Psychology: Learning, Memory, \& Cognition, 20, 443-460.

Elio, R, \& ANDERSON, J. R. (1981). The effects of category generalizations and instance similarity on schema abstraction. Journal of Experimental Psychology: Human Learning \& Memory, 7, 397-417.

ERICKSON, M. A., \& KruschKe, J. K. (1998). Rules and exemplars in category learning. Journal of Experimental Psychology: General, 127, 107-140.

GARNER, W. R. (1974). The processing of information and structure. Hillsdale, NJ: Erlbaum.

Gentner, D., \& Markman, A. B. (1994). Structural alignment in comparison: No difference without similarity. Psychological Science, $\mathbf{5}$ 152-158.

GoldSTONE, R. L. (1996). Isolated and interrelated concepts. Memory \& Cognition, 24, 608-628.

Goodman, N. (1983). Fact, fiction, and forecast (4th ed.). Cambridge, MA: Harvard University Press. (Original work published 1954)

INHELDER, B., \& PIAGET, J. (1964). The early growth of logic in the child. New York: Norton.

KAPLAN, A. (1999). Alignability and prior knowledge in category learning. Unpublished doctoral dissertation, University of Illinois, UrbanaChampaign.

Kersten, A. \& Billman, D. (1997). Event category learning. Journal of Experimental Psychology: Learning, Memory, \& Cognition, 23, 638658.

Kersten, A., Goldstone, R. L., \& Schaffert, A. (1998). Two competing attentional mechanisms in category learning. Journal of Experimental Psychology: Learning, Memory, \& Cognition, 24, 1437-1458.

KornBLITH,H. (1995). Our native inferential tendencies. In A. I. Goldman (Ed.), Readings in philosophy and cognitive science (pp. 69-94). Cambridge, MA: MIT Press.

KRUSCHKE, J. K. (1990). ALCOVE: A connectionist model of category learning (Cognitive Science Tech. Rep. No. 19). Bloomington:Indiana University.

KruschKe, J. K. (1992). ALCOVE: An exemplar-based connectionist model of category learning. Psychological Review, 99, 22-44.

KRUSCHKE, J. K. (1993). Three principles for models of category learning. In G. V. Nakamura, R. Taraban, \& D. L. Medin (Eds.), The psychology of learning and motivation (Vol. 29, pp. 57-90). San Diego: Academic Press.

Lassaline, M. E., \& Murphy, G. L. (1998). Alignment and category learning. Journal of Experimental Psychology: Learning, Memory, \& Cognition, 24, 144-160.

Macario, J. F., Shipley, E. F., \& Billman, D. (1990). Induction from a single instance: Formation of a novel category. Journal of Experimental Child Psychology, 50,179-199.

Macromind (1992). Director [Computer program]. San Francisco: Author.

Malt, B. C., \& Smith, E. E. (1983). Correlated properties in natural categories. Journal of Verbal Learning \& Verbal Behavior, 8, 828835.

Martin, J., \& Billman, D. (1991). Variability bias and category learning. In L. A. Birnbaum \& G. C. Collins (Eds.), Machine learning: Proceedings of the Eighth International Workshop (ML91) (pp. 90-94). San Mateo, CA: Morgan Kaufmann.
Medin, D. L., Altom, M. W., Edelson, S. M., \& Freko, D. (1982). Correlated symptoms and simulated medical classification. Journal of Experimental Psychology: Learning, Memory, \& Cognition, 8, 37-50.

Medin, D. L., \& Schaffer, M. M. (1978). A context theory of classification learning. Psychological Review, 85, 207-238.

Medin, D. L., \& Thau, D. M. (1992). Theories, constraints, and cognition. In H. L. Pick, P. van den Broek, \& D. C. Knill (Eds.), Cognition: Conceptual and methodological issues (pp. 165-187). Washington, DC: American Psychological Association.

Merriman, W. F. (1999). Competition, attention, and young children's lexical processing. In $\mathrm{B}$. MacWhinney (Ed.), The emergence of language (pp. 331-356). Hillsdale, NJ: Erlbaum.

Murphy, G. L., \& Allopenna, P. D. (1994). The locus of knowledge effects in concept learning. Journal of Experimental Psychology: Learning, Memory, \& Cognition, 19, 904-919.

Murphy, G. L., \& Medin, D. L. (1985). The role of theories in conceptual coherence. Psychological Review, 92, 289-316.

MuRPHY, G. L., \& WISNIEWSKI, E. J. (1989). Feature correlations in conceptual representations. In G. Tiberghien (Ed.), Advances in cognitive science: Vol 2. Theory and application (pp. 23-45). Chichester, U.K.: Ellis Harwood.

Nisbett, R. E., Krantz, D. H., Jepson, C., \& Kunda, Z (1983). The use of statistical heuristics in everyday inductive reasoning. Psychological Review, 90, 339-363.

NosOFSKY, R. M. (1984). Choice, similarity and the context model of classification. Journal of Experimental Psychology: Learning, Memory, \& Cognition, 10, 104-114.

Nosofsky, R. M., \& PAlmeri, T. J. (1998). A rule-plus-exception model for classifying objects in continuous-dimension spaces. Psychonomic Bulletin \& Review, 5, 345-369.

Nosofsky, R. M., Palmeri, T. J., \& McKinley, S. C. (1994). Rule-plusexception model of classification learning. Psychological Review, 101, 53-79.

Rehder, B., \& Hastie, R. (2001). Causal knowledge and categories: The effects of causal beliefs on categorization, induction, and similarity. Journal of Experimental Psychology: General, 130, 323-360.

Rosch, E. H. (1978). Principles of categorization. In E. H. Rosch \& B. B. Lloyd (Eds.), Cognition and categorization (pp. 27-48). Hillsdale, NJ: Erlbaum.

RuSSELL, S. J. (1986). Preliminary steps toward the automation of induction. In AAAI-86 Fifth National Conference on Artificial Intelligence (pp. 477-484). Philadelphia: American Association for Artificial Intelligence. Los Altos, CA: Morgan Kaufman.

Shipley, E. F. (1993). Categories, hierarchies, and induction. In D. L. Medin (Ed.), The psychology of learning and motivation (Vol. 30, pp. 265-301). San Diego: Academic Press.

Sifonis, C., \& Ross, B. (1999, August). Alignment effects on learning the use of categories. Poster presented at the meeting of the Cognitive Science Society, Vancouver, BC.

Trabasso, T., \& Bower, G. H. (1968). Attention in learning. New York: Wiley.

VICENTE, K. J. (1990). Coherence- and correspondence-driven work domains: Implications for system design. Behaviour \& Information Technology, 9, 493-502.

WisniewsKi, E. J., \& Medin, D. L. (1994). On the interaction of theory and data in concept learning. Cognitive Science, 18, 221-281.

YAMAUCHI, T., \& MARKMAN, A. B. (2000). Learning categories composed of varying instances: The effect of classification, inference, and structural alignment. Memory \& Cognition, 28, 64-78. 


\section{APPENDIX \\ Similarity Control for Experiment 2}

Similarity relations were organized to favor the inconsistent contrast stimuli over the consistent contrast stimuli. Similarity accounts would predict that many pairs of instance matching on many attributes within the same category would facilitate learning and that, conversely, few pairs of instances matching on many attributes in different categories would facilitate learning.

In controlling similarity, we favored the inconsistent contrast condition, according to a wide class of similarity metrics. This class includes any metric in which similarity is a fixed, monotonically increasing function of the number (and degree) of matching attributes and in which increases of the number of matching attributes at high levels of similarity have an influence equivalent to or greater than that from increases at low levels of similarity. Although the total number of matches may be important (additive metrics), there is considerable evidence that people are more influenced by a few highly similar items than by multiple low-similarity items (multiplicative metrics; Medin \& Shaffer, 1978).
Therefore, we favored the inconsistent contrast condition according to the total number of feature matches summed across instance pairs (within category, sum of matches $=58$ for consistent contrast and 60 for inconsistent contrast; between category, sum of matches $=61$ for consistent contrast and 48 for inconsistent contrast). We also particularly favored it according to the number of very similar pairs (within category, 8 pairs matched on 4 attributes for consistent contrast and 12 pairs matched on 4 attributes for inconsistent contrast; between category, 5 pairs matched on 4 attributes for consistent contrast and 0 pairs matched on 4 attributes for inconsistent contrast).

In summary, the inconsistent condition had no misleading, highly similar between-category pairs (none matched on 3 or 4 attributes), whereas the consistent condition had 5 highly similar between-category pairs (matching on 4 attributes).

(Manuscript received May 12, 2000; revision accepted for publication April 2, 2001.) 